\title{
Mechanical Insufflation-Exsufflation Versus Conventional Chest Physiotherapy in Children With Cerebral Palsy
}

\author{
Rasintra Siriwat MD, Jitladda Deerojanawong MD, Suchada Sritippayawan MD, \\ Sumalee Hantragool MD, and Prapasri Cheanprapai RN
}

\begin{abstract}
BACKGROUND: The cough mechanism is often impaired in children with quadriplegic spastic cerebral palsy, accounting for the high prevalence of pneumonia and atelectasis requiring prolonged hospitalization. Conventional chest physiotherapy (CPT) is a current technique recommended at the onset of lower-respiratory infections in cerebral palsy. Previous studies have demonstrated the usefulness of mechanical insufflation-exsufflation (MI-E) in children with neuromuscular disease. To date, there has been no study of MI-E in children with quadriplegic spastic cerebral palsy. The objective of the study is to compare the efficacy in reducing hospital stay and improvement of atelectasis between MI-E and CPT in children with quadriplegic spastic cerebral palsy with lower-respiratory infections. METHODS: This study is a randomized controlled trial. Children with quadriplegic spastic cerebral palsy, age 6 months to $18 \mathrm{y}$, admitted for lowerrespiratory infections and/or atelectasis at King Chulalongkorn Memorial Hospital between June 1, 2014, and March 31, 2015, were recruited. Those with pneumothorax, severe pneumonia, active tuberculosis, and shock were excluded. Children were randomized into the MI-E or CPT group. The MI-E group received MI-E (3 therapies/d), and the CPT group received CPT (1 therapy/d). Vital signs per protocol and chest radiograph as needed were recorded. RESULTS: There were 22 children enrolled in the study, 11 in the MI-E and 11 in the CPT group. Demographic data were comparable in both groups. The length of hospital stay was similar in both groups (MI-E 4-24 d vs CPT 6-42 d, $P=.15$ ). There were 17 subjects with atelectasis (MI-E $[n=9]$ versus CPT $[n=8]$ ). In this atelectasis subgroup, MI-E had shortened therapy time when compared with CPT $(2.9 \pm 0.8 \mathrm{~d}$ vs $3.9 \pm 0.6 \mathrm{~d}, P=.01)$. No complications were observed. CONCLUSIONS: MI-E is proven to be beneficial in shortening the duration of airway clearance in children with quadriplegic spastic cerebral palsy presenting with lowerrespiratory infections and atelectasis. MI-E is a safe and efficient intervention for airway clearance. Key words: mechanical insufflation-exsufflation; cough assist; children; cerebral palsy; conventional chest physiotherapy; airway clearance; atelectasis. [Respir Care 2018;63(2):187-193. (C 2018 Daedalus Enterprises]
\end{abstract}

\section{Introduction}

Cough is a complex activity that aids the airway clearance process, assists in removing excessive pulmonary

The authors are affiliated with the Department of Pediatrics, Faculty of Medicine, Chulalongkorn University, Bangkok, Thailand.

Dr Siriwat presented a version of this work at the Thai Congress of Pediatrics annual meeting, held October 15, 2015, in Bangkok, Thailand.

The authors have disclosed no conflicts of interest.

Correspondence: Jitladda Deerojanawong MD, Division of Pulmonary and Critical Care, Department of Pediatrics, Faculty of Medicine, Chu- secretions from airways, resolves atelectasis, and protects against aspiration. The cough mechanism has 3 phases: inspiratory, compressive, and expiratory. ${ }^{1}$ Each phase of this cough physiology may be lacking in children with neurological impairment, including cerebral palsy. Children with quadriplegic spastic cerebral palsy have an ineffective cough due to dystonia and poor coordination. They have decreased ability to close the glottis and often

\footnotetext{
lalongkorn University, 1873 Rama IV Road, Patumwan, Bangkok 10330, Thailand. E-mail: jitladda.d@ chula.ac.th.
}

DOI: $10.4187 /$ respcare. 05663 
have chronic aspiration, which leads to diminished sensitivity to cough. Most importantly, this group of patients tend to have scoliosis and decreased mobility, which contributes to poor ventilation, which then causes atelectasis, inadequate clearance of lower-airway secretions, particularly during respiratory infections, and prolongs hospitalization. ${ }^{2}$ Conventional chest physiotherapy (CPT), including chest percussion, vibration, postural drainage, and manually assisted cough, should be provided daily during respiratory infections. ${ }^{2-4}$

Children with quadriplegic spastic cerebral palsy may be unable to endure manual CPT because of thoracic deformity, cognitive impairment, and inability to cooperate. Additionally, manual techniques are also time-consuming and require specially trained clinicians. ${ }^{5}$ Several methods have been developed to increase effective cough, including intrapulmonary percussion ventilation and high-frequency chest wall compression. ${ }^{6,7}$ Cough augmentation with mechanical insufflation-exsufflation (MI-E) was introduced in the 1950s as an effective airway clearance modality in adults with poliomyelitis. ${ }^{8}$ MI-E works by regularly administering positive pressure to augment inspiration and then negative pressure to enhance expiratory flow. ${ }^{9,10}$ The device is effective at increasing cough peak flow for patients with reduced or no capacity to cough and expectorate. ${ }^{11}$

Early experiences with MI-E have been effective in adults with neuromuscular weakness, ${ }^{12}$ including Duchenne muscular dystrophy ${ }^{13}$ and amyotrophic lateral sclerosis. ${ }^{14}$ Research on the device has also provided good tolerance and physiologic improvement in patients with both restrictive and obstructive disease. ${ }^{15}$ Moreover, MI-E has been successful with an artificial interface. For example, Sancho et $\mathrm{al}^{16}$ established beneficial use of MI-E in unconscious or sedated subjects with less irritation, less pain, less tiring, and more comfort than conventional suctioning via tracheostomy tube. ${ }^{10}$

Since its introduction to pediatric practice, MI-E studies have been limited. In 2000 , Bach et al ${ }^{17}$ found that using MI-E for airway clearance in spinal muscular atrophy type 1 can avoid tracheostomy throughout early childhood. One prior study found a reduction in the frequency of pneumonias with resolved chronic atelectasis after using MI-E in children with neuromuscular disease. ${ }^{12}$ Later, a short-term randomized cross-over study found that the addition of MI-E with CPT shortened airway-clearance sessions compared with CPT alone in subjects with neuromuscular disease and upperrespiratory-tract infection. ${ }^{18}$ To date, there have been no MI-E safety and efficacy studies for airway clearance in children with cerebral palsy. The purpose of this study was to determine efficacy, in terms of reducing hospital stay and improvement of atelectasis, of MI-E in chil-

\section{QUICK LOOK}

\section{Current knowledge}

Mechanical insufflation-exsufflation (MI-E) delivers a positive-pressure insufflation followed by an expulsive exsufflation, thereby simulating a normal cough. MI-E has been described as an efficient technique for cough augmentation in patients with neuromuscular diseases, through either a facial mask or an endotracheal tube.

\section{What this paper contributes to our knowledge}

The use of MI-E in children with quadriplegic spastic cerebral palsy could shorten the duration of airway clearance in those hospitalized with respiratory-tract infection and atelectasis. This device appears to be safe, well tolerated and may be a good alternative intervention for airway clearance. However, the cost of the device should be considered.

dren with quadriplegic spastic cerebral palsy who were admitted with respiratory-tract infection.

\section{Methods}

A prospective randomized control trial was performed in children with quadriplegic spastic cerebral palsy, age 6 months to $18 \mathrm{y}$, who were admitted with acute respiratory-tract infection to the Department of Pediatrics, King Chulalongkorn Memorial Hospital during 2014-2015. The study was approved by the institutional review board (approval 241/57), and all subjects and/or parents gave informed consent. All study subjects who were consulted for secretion clearance or chest physiotherapy were recruited. Patients who presented with pneumothorax, rapidly progressive chest infection, decreased oxygen saturation $<92 \%$ even after oxygen supplementation more than $\mathrm{F}_{\mathrm{IO}_{2}}$ 0.6 , and those who had contagious diseases were excluded. This trial was registered with Thai Clinical Trial Registration number TCTR20170412001.

Each eligible subject was randomly assigned to receive either one of 2 treatments by a computerized mixed-box method. The MI-E group received MI-E (Cough Assist E70, Philips Respironics, Murrysville, Pennsylvania) 3 times/d. Our MI-E protocol involved 3-5 cycles with insufflation/exsufflation pressure beginning with $+15 \mathrm{~cm} \mathrm{H}_{2} \mathrm{O}$ to $-15 \mathrm{~cm} \mathrm{H}_{2} \mathrm{O}$ and maximum pressure of $+40 \mathrm{~cm} \mathrm{H}_{2} \mathrm{O}$ to $-40 \mathrm{~cm} \mathrm{H}_{2} \mathrm{O}$. Insufflation and exsufflation time were each 2-3 s. Pressure for each subject was adjusted according to amount of secretions, tolerance, and chest auscultation during each session. Rest periods were provided for suctioning secre- 
tions from mouth, nose, or tracheostomy stoma. The numbers of total sets were $4-6$ with duration between 20 and $30 \mathrm{~min}$ as guided by subject fatigue and comfort. At the beginning, the machine was used in manual mode to find the appropriate pressure. Then, for the second treatment, auto mode was set, and pressure was used until stopping treatment. All of the treatments were performed by an experienced researcher and respiratory nurse.

The CPT group received conventional chest physiotherapy by a physiotherapist once per day, which consisted of a cycle of chest percussion, vibration, postural drainage, and manual assisted cough. In subjects with atelectasis, nebulization via EzPAP (Smiths Medical, Minneapolis, Minnesota) was used for helping lung expansion. Bronchodilators were considered in subjects with wheezing, and normal saline was used in subjects found to have sticky sputum. The EzPAP system was set with the flow beginning at $5 \mathrm{~L} / \mathrm{min}$, which was increased until the desired PAP was reached. A small-volume nebulizer with 6-8 L/min of $100 \%$ oxygen was connected to the EzPAP. The desired PAP was considered using a pressure gauge for monitoring the expiratory pressure along with equally good air entry in both lungs by chest auscultation. The EzPAP was connected via a face mask for children. Suction was indicated and performed when secretions were noticed through the mouth and nose or per tracheostomy tube.

Following both types of treatment, antibiotics, oxygen, bronchodilators, or normal saline nebulization and fluid replacement therapy were appropriately considered by the caregiver. Throughout each treatment session, heart rate and $\mathrm{S}_{\mathrm{pO}_{2}}$ were recorded. Blood pressure was measured before and after each treatment to ensure clinical steadiness. Auscultation and clinical assessment were also used to monitor any complications. A chest radiograph was obtained for every subject on the first day of admission with additional chest radiographs dependent on clinically changes of subjects.

Termination of MI-E or chest physiotherapy was considered when subjects met all of the following criteria: body temperature and oxygen saturation normal and stable and signs of minimal sputum production with resolution of abnormal chest auscultation and chest radiograph.

Outcomes were defined as follows. Hospital stay was the time from the first day of admission until the subject was discharged from the hospital, and duration of treatment time was the time from the first day of treatment until the subject met the criteria for termination of MI-E or chest physiotherapy.

\section{Statistical Analysis}

Sample size was calculated based on the data obtained from our pilot study. We used mean duration of

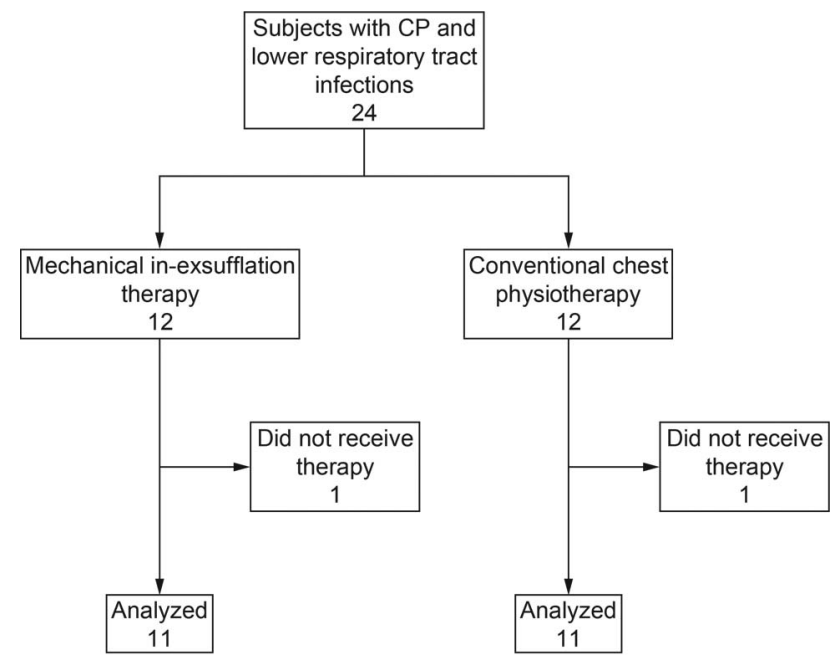

Fig. 1. Flow chart. $\mathrm{CP}=$ cerebral palsy.

hospital stay as the main measurement for sample size calculation. Children with cerebral palsy who received conventional chest physiotherapy alone had a mean hospital stay of $8 \pm 2.58 \mathrm{~d}$. We determined that a sample size of 38 (19 in each group) would provide the study with $80 \%$ power with an $\alpha$ level of 0.05 to detect a $30 \%$ decrease in the mean length of hospital stay in subjects who received MI-E.

All normally distributed data are expressed as mean \pm SD. Non-normally distributed data are expressed as median and interquartile range. Differences between the 2 groups (CPT and MI-E) were analyzed using parametric tests or nonparametric tests. For comparison of 2 means, a Student paired $t$ test analysis was used. Student $t$ tests or Mann-Whitney U-tests were considered appropriate for continuous variables, and the chi-square or Fisher exact test was used for categorical data. To determine whether there was an impact of time since initiation of treatment on the change in physiological variables, a multilevel mixed-effects linear regression model was used. $P<.05$ was considered statistically significant. All data were analyzed with Stata 12 (StataCorp, College Station, Texas).

\section{Results}

An interim analysis was completed earlier after enrollment of 22 subjects with quadriplegic spastic cerebral palsy. All had lower-respiratory-tract infection and required airway clearance therapy. Subject age ranged from 7 months to $12 \mathrm{y}$ (median age $6.4 \mathrm{y}$ ), $>70 \%$ had a tracheostomy tube, and the majority of chest radiography in this study showed atelectasis. There were a total of 22 subjects analyzed, 11 in the MI-E group and 11 in the CPT group (Fig. 1). Baseline clinical characteristics were comparable 
Table 1. Clinical Characteristics of Study Patients

\begin{tabular}{lccr}
\hline \hline \multicolumn{1}{c}{ Demographic Data } & MI-E $(n=11)$ & CPT $(n=11)$ & $P$ \\
\hline Age, mean \pm SD y & $6.6 \pm 3.5$ & $6.2 \pm 4.1$ & .65 \\
Male sex, $n(\%)$ & $3(27.3)$ & $4(36.4)$ & $>.99$ \\
Weight, mean \pm SD kg & $21.2 \pm 7.7$ & $17.9 \pm 8.4$ & .96 \\
Height, mean \pm SD m & $110.5 \pm 21.0$ & $100.5 \pm 22.6$ & .30 \\
BMI, mean \pm SD kg/m ${ }^{2}$ & $16.7 \pm 2.0$ & $16.6 \pm 2.6$ & .89 \\
Underlying disease, $n(\%)$ & & & \\
$\quad$ GERD & $11(100)$ & $11(100)$ & $>.99$ \\
$\quad$ Tracheomalacia & 0 & $1(9.1)$ & $>.99$ \\
Tracheostomy tube, $n(\%)$ & $8(72.7)$ & $8(72.7)$ & $>.99$ \\
Home oxygen, $n(\%)$ & $9(81.8)$ & $8(72.7)$ & \\
Home ventilator, $n(\%)$ & 0 & $1(9.1)$ & \\
& & & \\
MI-E $=$ mechanical insufflation-exsufflation & & & \\
CPT $=$ conventional chest physiotherapy & & & \\
BMI $=$ body mass index & & & \\
GERD $=$ gastroesophageal reflux disease & & & \\
\hline
\end{tabular}

Table 2. Physiologic Data

\begin{tabular}{|c|c|c|c|}
\hline Demographic Data & MI-E $(n=11)$ & $\mathrm{CPT}(n=11)$ & $P$ \\
\hline Body temperature, mean $\pm \mathrm{SD}{ }^{\circ} \mathrm{C}$ & $37.7 \pm 0.5$ & $37.4 \pm 0.4$ & .17 \\
\hline $\begin{array}{l}\text { Breathing frequency, mean } \pm \mathrm{SD} \\
\text { breaths/min }\end{array}$ & $35.8 \pm 9.9$ & $32.5 \pm 7.7$ & .40 \\
\hline Pulse rate, mean $\pm \mathrm{SD}$ pulse $/ \mathrm{min}$ & $102.6 \pm 17.5$ & $107.9 \pm 11.4$ & .41 \\
\hline Blood pressure, mean $\pm \mathrm{SD} \mathrm{mm} \mathrm{Hg}$ & $108 \pm 4.0$ & $106.6 \pm 3.3$ & .68 \\
\hline $\mathrm{S}_{\mathrm{pO}_{2}}$, mean $\pm \mathrm{SD} \% *$ & $98.4 \pm 1.5$ & $98.6 \pm 1.8$ & .80 \\
\hline Chest retraction, $n(\%)$ & $11(100)$ & $8(72.7)$ & .21 \\
\hline \multicolumn{4}{|l|}{ Breath sounds, $n(\%)$} \\
\hline Decreased & $4(36.4)$ & $3(27.3)$ & $>.99$ \\
\hline Crepitation & 7 (63.6) & $8(72.7)$ & $>.99$ \\
\hline \multicolumn{4}{|l|}{$\begin{array}{l}\text { * Baseline of individual oxygen supplementation. } \\
\text { MI-E = mechanical insufflation-exsufflation } \\
\text { CPT = conventional chest physiotherapy }\end{array}$} \\
\hline
\end{tabular}

in both groups $(P>.05)$ (Table 1$)$. There was no significant difference in physical measurement data between both groups in terms of body temperature, breathing frequency, heart rate, blood pressure, pulse oximetry and breath sound (Table 2). Our daily recorded data showed that all breathing frequencies, heart rates, and $\mathrm{S}_{\mathrm{pO}_{2}}$ levels were improved in both groups after $4 \mathrm{~d}$, without a statistical difference between the groups. The breathing frequency in the MI-E group was slightly higher than the CPT group throughout the study (no statistical significance) $(P=.32)$ (Fig. 2$)$.

Subjects in the CPT group had a slightly longer length of hospital stay compared with those in the MI-E group but with no statistical difference $(P=.15)$. Additionally, there were no differences in duration of therapy time $(P=.056)$ and days of oxygen used in either group $(P=.62)$ (Table 3). All of the repeated chest radiographs were improved in both groups after treatment ( 9 in the MI-E group and 8 in the CPT group). All chest radiographs were in-

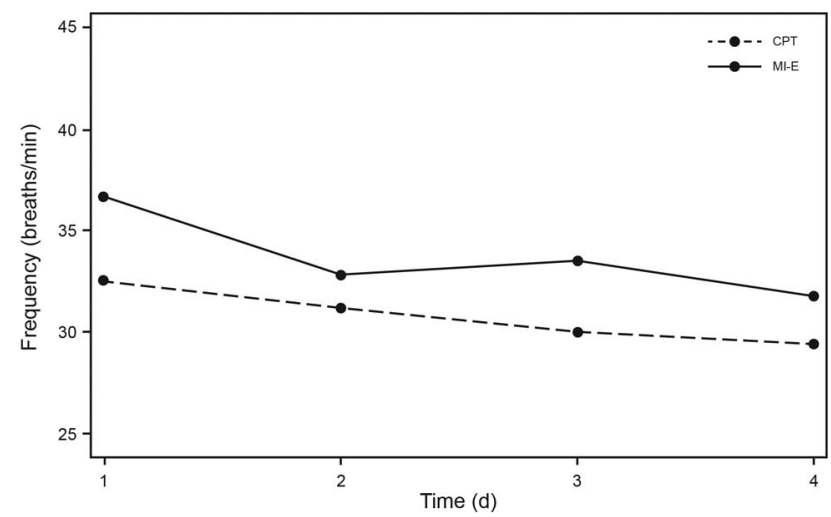

Fig. 2. Comparison of breathing frequency between the mechanical in-exsufflation (MI-E) group and conventional chest physiotherapy (CPT) group. $P=.32$.

Table 3. Comparison of Outcomes in Both Groups

\begin{tabular}{lccc}
\hline \hline \multicolumn{1}{c}{ Data } & MI-E $(n=11)$ & CPT $(n=11)$ & $P$ \\
\hline $\begin{array}{l}\text { Hospital length of stay, median } \\
\text { (range) d }\end{array}$ & $9(4-24)$ & $12(6-42)$ & .15 \\
$\begin{array}{l}\text { Oxygen used, median (range) d } \\
\text { Therapy time, mean } \pm \text { SD d }\end{array}$ & $5(1-12)$ & $5(1-10)$ & .62 \\
& $3 \pm 0.77$ & $3.72 \pm 0.90$ & .056 \\
$\begin{array}{l}\text { MI-E = mechanical insufflation-exsufflation } \\
\text { CPT = conventional chest physiotherapy }\end{array}$ & & & \\
\hline
\end{tabular}

Table 4. Comparison of Outcomes in the Atelectasis Subgroup

\begin{tabular}{lccr}
\hline \hline \multicolumn{1}{c}{ Outcomes } & $\begin{array}{c}\text { MI-E } \\
(n=9)\end{array}$ & $\begin{array}{c}\text { CPT } \pm \text { EzPAP } \\
(n=8)\end{array}$ & $P$ \\
\hline $\begin{array}{l}\text { Hospital length of stay, median } \\
\text { (range) d }\end{array}$ & $9(4-24)$ & $12.5(6-42)$ & .12 \\
$\begin{array}{l}\text { Oxygen used, median (range) d } \\
\text { Therapy time, mean } \pm \text { SD d }\end{array}$ & $5(2-12)$ & $6.5(4-10)$ & .77 \\
Improvement of atelectasis, $n(\%)$ & $9(100)$ & $8.9 \pm 0.6$ & .01 \\
& & 8.99
\end{tabular}

MI-E = mechanical insufflation-exsufflation

$\mathrm{CPT}=$ conventional chest physiotherapy

terpreted by a pediatric radiologist who was blinded to group distribution.

Significant differences were shown in a subgroup of subjects with atelectasis. The MI-E group had significantly shortened therapy time when compared with CPT $(P=.01)$. In this atelectasis subgroup, hospital stay and days of oxygen use were similar in both groups (Table 4).

From this study, the mean pressure and other parameters used in MI-E were observed as follows: Insufflation/exsufflation pressures (mean $\pm \mathrm{SD}$, range) were $35 \pm 7.1$ $(20-40) / 35 \pm 7.1(20-40) \mathrm{cm} \mathrm{H}_{2} \mathrm{O}$, and insufflation/exsufflation times were $2 / 3 \mathrm{~s}$ with oscillations of $20 \mathrm{~Hz}$ and amplitude of $5 \mathrm{~cm} \mathrm{H}_{2} \mathrm{O}$. There were no episodes of pneu- 
mothorax or cardiopulmonary compromise. One subject had mild symptoms of gastroesophageal reflux.

\section{Discussion}

This randomized control trial study compared the efficacy of MI-E with CPT in children with quadriplegic spastic cerebral palsy who had lower-respiratory-tract infections. Key outcome measures were hospital length of stay and improvement of atelectasis. Previous research has demonstrated that the use of MI-E has been broadly accepted in adults with neuromuscular disease. In children, there are limited data, but most studies have found benefits of using MI-E in children with neuromuscular disease. ${ }^{12,18}$ To our knowledge, no studies have compared current CPT practices (manual percussion, vibration, postural drainage, and manual assisted cough) with MI-E in children with quadriplegic spastic cerebral palsy.

Our results showed no differences between the groups for duration of hospital stay. However, our study had a small number of subjects and was unable to exclude factors influencing hospital stay. To better understand this, we observed that after subjects had improved clinical status and met criteria for termination of using MI-E or CPT treatment, their hospital stay was extended due to other reasons, including duration of intravenous antibiotics and unavailability of parents to have their children discharged. Moreover, our results found no difference in therapy time and days of oxygen used in both groups.

When secondary outcomes were examined, results revealed that there is a role for MI-E in assisting with secretion clearance. Subjects with atelectasis had reduced therapy time when compared with subjects who received routine CPT combined with nebulization via EzPAP, although it was only a 1-d difference, and this may lack clinical importance. Most subjects in our study showed evidence of lung collapse. We explain this finding by increased secretions, leading to bronchial obstruction, ineffective cough, and immobilization of subjects with quadriplegic spastic cerebral palsy. This finding is comparable with the previous study reported by Chatwin et al, ${ }^{18}$ which found that the addition of MI-E with current respiratory physiotherapy shortened treatment time compared with respiratory physiotherapy alone in noninvasive ventilationdependent neuromuscular disease. However, there were some differences related to defining treatment time: Our study analyzed days, whereas the study by Chatwin et al ${ }^{18}$ analyzed minutes. These authors also did not study an atelectasis subgroup. In our results, we hypothesized that a decrease in days of treatment represented rapid improvement of lung function and atelectasis. Results from our study were similar to those of prior studies on the improvement in atelectasis when using MI-E as an airway clearance technique. In their study, Miske et $\mathrm{al}^{12}$ found clearing of chronic atelectasis in children with neuromuscular disease after beginning MI-E and reported rapid improvement of atelectasis in a 22-month-old with spinal muscular atrophy type 1 after using MI-E. Importantly, no prior studies were reported or described using MI-E in children with quadriplegic spastic cerebral palsy with atelectasis. This result is probably due to MI-E clearing secretions by simulating a natural cough as mentioned above.

From our observations, MI-E is a safe and effective method of alternative airway clearance in subjects with artificial airways. A similar study was conducted by Sancho et al. ${ }^{16}$ They found that $\mathrm{S}_{\mathrm{pO}_{2}}$, peak inspiratory pressure, mean airway pressure, and work of breathing performed by the ventilator were improved after using MI-E via a tracheostomy tube compared with conventional tracheal suctioning. ${ }^{10,16}$

All of the subjects in our study were discharged home without any severe complications from either therapy. In previous reports, complications of MI-E use in adults were rare. These included nausea, bradycardia, tachycardia, and abdominal distention. ${ }^{19,20}$ In our series, only one subject reported esophageal reflux. The rapid decrease in intrathoracic pressure below intra-abdominal pressure associated with negative pressure exsufflation could potentially predispose patients to gastroesophageal reflux with aspiration, especially in patients with gastroesophageal reflux susceptibility, as found in our population. Timing the intervention is very important, and we recommend at least a 2-h window after feeding.

As mentioned above, the mean insufflation/exsufflation pressure was found to be higher in our study than in prior studies. ${ }^{15,18}$ The higher pressure was explained by the resistance from artificial airways. Guérin et al, ${ }^{21}$ in their lung model study, found that the artificial airway significantly reduced peak expiratory flow during therapy with MI-E which resulted from the narrow inner diameter of the artificial airway. Accordingly, higher pressure is used in patients with artificial airways $\left(>30 \mathrm{~cm} \mathrm{H}_{2} \mathrm{O}\right)$. Striegl et al ${ }^{22}$ studied a simulated infant lung model with a tracheostomy tube and suggested that effective pressure in maximizing inspiratory volume and expiratory flow were an insufflation pressure of $20-30 \mathrm{~cm} \mathrm{H}_{2} \mathrm{O}$ and an exsufflation pressure of -30 to $-40 \mathrm{~cm} \mathrm{H}_{2} \mathrm{O}$.

In the present study, it is important to note that MI-E was used in manual mode at the time of the first therapy to adjust appropriate pressures as mentioned in the Methods. Auto mode was then set accordingly, and pressure was kept constant until subjects met the criteria for termination of therapy. This method differed from previous studies that used manual mode throughout the study. ${ }^{12,15,18}$ From our experience, using auto mode was easy, saved time, and resulted in fewer complications. Additionally, mechanical insufflation-exsufflation (Cough Assist E70) has an oscillation mode. We considered using oscillation in both insufflation and exsufflation to better encourage expectoration of secretions from the air- 
way. Oscillation settings were constant with $20-\mathrm{Hz}$ frequency and 5- $\mathrm{cm} \mathrm{H}_{2} \mathrm{O}$ amplitude. To our knowledge, no previous studies have compared standard MI-E with MI-E with oscillation. However, we found no complications and observed more benefits from this mode.

In the study, we specificity wanted to determine whether using MI-E could reduce hospital length of stay and days of therapy time compared with routine chest physiotherapy, but we did not record the exact duration of time by minutes or hours in each therapy (both CPT and MI-E). In terms of the CPT group, children who had secretion retention were enrolled into a physiotherapy program by a physiotherapist at King Chulalongkorn Memorial Hospital, and therapy was performed daily. This program was limited by the small number of pediatric physiotherapists and a large burden of work. In MI-E therapy, to date there has been no standard protocol of MI-E for airway clearance or cough augmentation in children. For MI-E, general recommendations for routine use include 3-5 sets of 3-5 cycles each (of inspiration, exhalation, and pause) to be performed twice daily, which may be increased in frequency when unwell. We therefore decided to use MI-E 3 times/d during a respiratory-tract infection.

We acknowledge that our study has some limitations. There was a small number of subjects with quadriplegic spastic cerebral palsy who were admitted to King Chulalongkorn Memorial Hospital with lower-respiratory-tract infection. Therefore, the trial was terminated before the enrollment of subjects was calculated in pretrial estimates. Nevertheless, our prospective randomized controlled trial is the first study to examine the effects of MI-E in children with quadriplegic spastic cerebral palsy who have a respiratory-tract infection. As mentioned above, there was a difference in treatment frequency between MI-E and CPT, and we believe our methods would be more robust if we provided the same frequency for both methods in future studies. We encountered issues in finding other objective measurements in this study, such as peak expiratory flow, cough peak flow, and lung function tests (eg, due to subjects who were unable to perform any respiratory tests). Although we conducted the comparison between 2 different methods, we did not compare with a non-treatment group in this population. Moreover, chest radiographs were difficult to conduct on a regular schedule, as there was high concern for radiation exposure. In this study, the use of MI-E and therapy assessment were conducted by respiratory practitioners. Researchers were not blinded, therefore possibly increasing the chance of bias. Additionally, the machine itself is expensive and not available in all centers. Further study is needed to identify more subjects, long-term outcomes, and cost-effectiveness from using MI-E in this group of subjects.

\section{Conclusions}

Our study was limited in sample size; however, both treatment methods appeared to be effective and safe. There was no significant difference in hospital length of stay between methods. MI-E could be beneficial in shortening the duration of airway clearance in patients with quadriplegic spastic cerebral palsy presenting with lower-respiratory infections and atelectasis. Regarding effectiveness, MI-E appears to be a good alternative intervention for airway clearance, showing fewer adverse effects and a reduction of labor hours. Future studies including a larger number of subjects and assessing long-term effects of MI-E are recommended.

\section{REFERENCES}

1. Chang AB. The physiology of cough. Paediatr Respir Rev 2006; $7(1): 2-8$.

2. Seddon PC, Khan Y. Respiratory problems in children with neurological impairment. Arch Dis Child 2003;88(1):75-78.

3. Fitzgerald DA, Follett J, Van Asperen PP. Assessing and managing lung disease and sleep disordered breathing in children with cerebral palsy. Paediatr Respir Rev 2009;10(1):18-24.

4. Balachandran A, Shivbalan S, Thangavelu S. Chest physiotherapy in pediatric practice. Indian Pediatr 2005;42(6):559-568.

5. Papastamelos C, Panitch HB, Allen JL. Chest wall compliance in infants and children with neuromuscular disease. Am J Respir Crit Care Med 1996;154(4 Pt 1):1045-1048.

6. Schramm CM. Current concepts of respiratory complications of neuromuscular disease in children. Curr Opin Pediatr 2000;12(3):203207.

7. Strickland SL, Rubin BK, Drescher GS, Haas CF, O'Malley CA, Volsko TA, et al. AARC clinical practice guideline: effectiveness of nonpharmacologic airway clearance therapies in hospitalized patients. Respir Care 2013;58(12):2187-2193.

8. Cherniack RM, Hildes JA, Alcock AJ. The clinical use of the exsufflator attachment for tank respirators in poliomyelitis. Ann Intern Med 1954;40(3):540-548.

9. Birnkrant DJ, Pope JF, Eiben RM. Management of the respiratory complications of neuromuscular diseases in the pediatric intensive care unit. J Child Neurol 1999;14(3):139-143.

10. Toussaint M. The use of mechanical insufflation-exsufflation via artificial airways. Respir Care 2011;56(8):1217-1219.

11. McCool FD, Rosen MJ. Nonpharmacologic airway clearance therapies: ACCP evidence-based clinical practice guidelines. Chest 2006; 129(1 Suppl):250S-259S.

12. Miske LJ, Hickey EM, Kolb SM, Weiner DJ, Panitch HB. Use of the mechanical in-exsufflator in pediatric patients with neuromuscular disease and impaired cough. Chest 2004;125(4):1406-1412.

13. Birnkrant DJ, Bushby KM, Amin RS, Bach JR, Benditt JO, Eagle M, et al. The respiratory management of patients with duchenne muscular dystrophy: a DMD care considerations working group specialty article. Pediatr Pulmonol 2010;45(8):739-748.

14. Miller RG, Jackson CE, Kasarskis EJ, England JD, Forshew D, Johnston W, et al. Practice parameter update: the care of the patient with amyotrophic lateral sclerosis: Drug, nutritional, and respiratory therapies (an evidence-based review): report of the quality standards subcommittee of the American Academy of Neurology. Neurology 2009;73(15):1218-1226.

15. Winck JC, Gonçalves MR, Lourenço C, Viana P, Almeida J, Bach JR. Effects of mechanical insufflation-exsufflation on respiratory 


\section{Mi-E vs CPT in Pediatric Cerebral Palsy}

parameters for patients with chronic airway secretion encumbrance. Chest 2004;126(3):774-780.

16. Sancho J, Servera E, Vergara P, Marín J. Mechanical insufflationexsufflation vs. tracheal suctioning via tracheostomy tubes for patients with amyotrophic lateral sclerosis: a pilot study. Am J Phys Med Rehabil 2003;82(10):750-753.

17. Bach JR, Niranjan V, Weaver B. Spinal muscular atrophy type 1: a noninvasive respiratory management approach. Chest 2000;117(4): 1100-1105.

18. Chatwin M, Simonds AK. The addition of mechanical insufflation/exsufflation shortens airway-clearance sessions in neuromuscular patients with chest infection. Respir Care 2009;54(11):1473-1479.
19. Bach JR. Update and perspective on noninvasive respiratory muscle aids: part 2: the expiratory aids. Chest 1994;105(5):1538-1544.

20. Homnick DN. Mechanical insufflation-exsufflation for airway mucus clearance. Respir Care 2007;52(10):1296-1305; discussion 13061307.

21. Guérin C, Bourdin G, Leray V, Delannoy B, Bayle F, Germain M, Richard JC. Performance of the coughassist insufflation-exsufflation device in the presence of an endotracheal tube or tracheostomy tube: a bench study. Respir Care 2011;56(8):1108-1114.

22. Striegl AM, Redding GJ, Diblasi R, Crotwell D, Salyer J, Carter ER. Use of a lung model to assess mechanical in-exsufflator therapy in infants with tracheostomy. Pediatr Pulmonol 2011;46(3):211-217. 